\title{
Beurling algebra analogues of theorems of Wiener-Lévy-Żelazko and Żelazko
}

by

\author{
S. J. Bhatt, P. A. DABhi and H. V. Dedania (Vallabh Vidyanagar)
}

\begin{abstract}
Let $0<p \leq 1$, let $\omega: \mathbb{Z} \rightarrow[1, \infty)$ be a weight on $\mathbb{Z}$ and let $f$ be a nowhere vanishing continuous function on the unit circle $\Gamma$ whose Fourier series satisfies $\sum_{n \in \mathbb{Z}}|\widehat{f}(n)|^{p} \omega(n)<\infty$. Then there exists a weight $\nu$ on $\mathbb{Z}$ such that $\sum_{n \in \mathbb{Z}}|\widehat{(1 / f)}(n)|^{p} \nu(n)$ $<\infty$. Further, $\nu$ is non-constant if and only if $\omega$ is non-constant; and $\nu=\omega$ if $\omega$ is nonquasianalytic. This includes the classical Wiener theorem $(p=1, \omega=1)$, Domar theorem ( $p=1, \omega$ is non-quasianalytic), Żelazko theorem $(\omega=1)$ and a recent result of Bhatt and Dedania $(p=1)$. An analogue of the Lévy theorem at the present level of generality is also developed. Given a locally compact group $G$ with a continuous weight $\omega$ and $0<p<1$, the locally bounded space $L^{p}(G, \omega)$ is closed under convolution if and only if $G$ is discrete if and only if $G$ admits an atom. This generalizes and refines another result of Żelazko.
\end{abstract}

Let $f$ be a continuous function on the unit circle $\Gamma$. Let $f$ have absolutely convergent Fourier series. The celebrated Wiener theorem [12] implies that if $f(z) \neq 0$ for all $z \in \Gamma$, then $1 / f$ has absolutely convergent Fourier series. Lévy's generalization [8] of Wiener's theorem implies that, for every function $\varphi$ holomorphic on some neighbourhood of the range of $f$, the function $\varphi \circ f$ has absolutely convergent Fourier series if so does $f$. Żelazko proved in [13] that both these theorems hold if absolute convergence is replaced by $p$ th power absolute convergence for $0<p<1$.

Let $\omega$ be a weight on $\mathbb{Z}$, that is, $\omega: \mathbb{Z} \rightarrow[1, \infty)$ satisfies $\omega(m+n) \leq$ $\omega(m) \omega(n)(m, n \in \mathbb{Z})$. A complex sequence $\left(\lambda_{n}\right)_{n \in \mathbb{Z}}$ is $\omega$-absolutely convergent if $\sum_{n}\left|\lambda_{n}\right| \omega(n)<\infty$.

Domar proved in [4, Theorem 2.11] that if $f$ has $\omega$-absolutely convergent Fourier series and is nowhere vanishing on $\Gamma$, then $1 / f$ has $\omega$-absolutely convergent Fourier series provided $\omega$ is non-quasianalytic in the sense that $\sum_{n \in \mathbb{Z}}(\log \omega(n)) /\left(1+n^{2}\right)<\infty$. On the other hand, given $f$ having $\omega$ absolutely convergent Fourier series, it is established in [2] that there ex-

2010 Mathematics Subject Classification: 42A16, 42A28, 46 J05.

Key words and phrases: Fourier series, Wiener theorem, Beurling algebra, commutative Banach algebra, $p$-Banach algebra, atom. 
ists a weight $\nu$ on $\mathbb{Z}$ such that $1 / f$ (and analogously $\varphi \circ f$ ) has $\nu$-absolutely convergent Fourier series.

We prove the following theorem that includes all these results, which also gives a $p$ th power analogue of Domar's theorem.

TheOrem 1. Let $0<p \leq 1$, let $\omega$ be a weight on $\mathbb{Z}$, and let $f \in C(\Gamma)$ have pth power $\omega$-absolutely convergent Fourier series.

(I) If $f(z) \neq 0(z \in \Gamma)$, then there exists a weight $\nu$ on $\mathbb{Z}$ such that

(i) $1 / f$ has pth power $\nu$-absolutely convergent Fourier series;

(ii) $\nu$ is non-constant if and only if $\omega$ is non-constant;

(iii) $\nu(n) \leq \omega(n)(n \in \mathbb{Z})$.

(II) If $\varphi$ is a holomorphic function on some neighbourhood of the range of $f$, then there exists a weight $\chi$ on $\mathbb{Z}$ such that

(i) $\varphi \circ f$ has pth power $\chi$-absolutely convergent Fourier series;

(ii) $\chi$ is non-constant if and only if $\omega$ is non-constant;

(iii) $\chi(n) \leq \omega(n)(n \in \mathbb{Z})$.

Corollary 1. Let $0<p \leq 1$, let $\omega$ be a non-quasianalytic weight on $\mathbb{Z}$, and let $f \in C(\Gamma)$ be nowhere vanishing. If $f$ has pth power $\omega$-absolulely convergent Fourier series, then $1 / f$ has pth power $\omega$-absolutely convergent Fourier series.

Analogous to Gel'fand's proof of the Wiener theorem [7, p. 33], which is based on Banach algebras, we shall use Gel'fand theory of $p$-Banach algebras developed by Żelazko in the framework of locally bounded Beurling algebras.

Let $\mathcal{A}$ be a (complex) algebra and let $0<p \leq 1$. Then a mapping $\|\cdot\|: \mathcal{A} \rightarrow \mathbb{R}$ is a $p$-norm on $\mathcal{A}$ if, for $x, y \in \mathcal{A}$ and for $\alpha \in \mathbb{C}$,

(i) $\|x\| \geq 0$ and $\|x\|=0$ if and only if $x=0$;

(ii) $\|x+y\| \leq\|x\|+\|y\|$;

(iii) $\|\alpha x\|=|\alpha|^{p}\|x\|$;

(iv) $\|x y\| \leq\|x\|\|y\|$.

If $\mathcal{A}$ is complete in the $p$-norm, then $(\mathcal{A},\|\cdot\|)$ is a $p$-Banach algebra. Among unital algebras, $p$-Banach algebras are precisely the complete locally bounded algebras [13, Theorem 2.3]. Given a continuous weight $\omega: G \rightarrow[1, \infty)$ on a locally compact group $G$ satisfying $\omega(s t) \leq \omega(s) \omega(t)(s, t \in G)$, let $L^{p}(G, \omega)$ be the set of all measurable functions $f: G \rightarrow \mathbb{C}$ such that

$$
\|f\|_{p, \omega}:=\int_{G}|f(t)|^{p} \omega(t) d m(t)=\int_{G}|f(t)|^{p} d m_{\omega}(t)<\infty,
$$

where $m$ is the left invariant Haar measure on $G$, and $d m_{\omega}=\omega d m$. Though a complete locally bounded space can be dual-less, i.e., have no non-zero continuous linear functionals (which could be a hurdle in construction of 
vector-valued integrals), Żelazko [13] constructed a functional calculus in $p$ Banach algebras; and an offshot of his Gel'fand theory is that a semisimple, commutative, complete locally bounded algebra has sufficiently many continuous linear functionals. For a discrete abelian group $G$ and for $0<p \leq 1$, the space $\ell^{p}(G)$ is a $p$-Banach algebra with convolution. In fact, Żelazko proved that, for a locally compact group $G$ and for $0<p<1$, the complete locally bounded space $L^{p}(G)$ is closed under convolution if and only if $G$ is discrete. The following theorem gives a Beurling algebra analogue of this.

Theorem 2. Let $0<p<1$, let $G$ be a non-compact, locally compact group, and let $\omega$ be a continuous weight on $G$. Then the following are equivalent.

(i) $L^{p}(G, \omega)$ is closed under convolution.

(ii) $G$ is discrete.

(iii) $L^{p}(G, \omega)$ admits a non-zero continuous linear functional.

(iv) The set of continuous linear functionals on $L^{p}(G, \omega)$ separates the points of $L^{p}(G, \omega)$.

(v) $G$ admits an atom.

(vi) $G$ admits sufficiently many atoms.

In this case, if $G$ is abelian or $\omega$ is symmetric, $L^{p}(G, \omega)$ is semisimple.

Note that, in general, it is not known whether $L^{1}(G, \omega)$ or $\ell^{p}(G, \omega)$ $(0<p<1)$ is semisimple or not [3, p. 175].

Rolewicz [11] has discussed multi-dimensional generalizations of WienerŻelazko and Lévy-Żelazko theorems. In the same spirit, it would be interesting to investigate multi-dimensional analogues of Domar's theorem [4, Theorem 2.11] as well as the theorem in [2].

Recall that a measurable set $E \subset G$ is an atom if $0<m(E)<\infty$, and, for any measurable set $F \subset E, m(F)=0$ or $m(F)=m(E)$. A Banach $*$-algebra $A$ is an $A^{*}$-algebra if it admits a $C^{*}$-norm (not necessarily complete).

Proof of Theorem 1. Let

$$
\ell^{p}(\mathbb{Z}, \omega):=\left\{\lambda=\left(\lambda_{n}\right):|\lambda|_{p, \omega}:=\sum_{n \in \mathbb{Z}}\left|\lambda_{n}\right|^{p} \omega(n)<\infty\right\} .
$$

Then $\ell^{p}(\mathbb{Z}, \omega)$ is a convolution algebra. It is a $p$-Banach algebra with the $p$-norm $|\cdot|_{p, \omega}$. Let $A_{p}(\omega)=\left\{g \in C(\Gamma): \widehat{g} \in \ell^{p}(\mathbb{Z}, \omega)\right\}$. It is a $p$-Banach algebra with the pointwise operations and the $p$-norm being $\|g\|_{p, \omega}=|\widehat{g}|_{p, \omega}$. Thus $g \in C(\Gamma)$ has $p$ th power $\omega$-absolulely convergent Fourier series if and only if $g \in A_{p}(\omega)$ if and only if $\widehat{g} \in \ell^{p}(\mathbb{Z}, \omega)$.

We claim that the Gel'fand space $\triangle\left(A_{p}(\omega)\right)$ of $A_{p}(\omega)$ is homeomorphic to the closed annulus $\Gamma(\omega)=\{z \in \mathbb{C}: \rho(2, \omega) \leq|z| \leq \rho(1, \omega)\}$, where

$$
\rho(1, \omega)=\inf \left\{\omega(n)^{1 / n}: n \geq 1\right\} \quad \text { and } \quad \rho(2, \omega)=\sup \left\{\omega(n)^{1 / n}: n \leq-1\right\} \text {. }
$$


For $z \in \Gamma(\omega)$, define

$$
\varphi_{z}(g)=\sum_{n \in \mathbb{Z}} \widehat{g}(n) z^{n} \quad\left(g \in A_{p}(\omega)\right) .
$$

Then, for large $n_{0}$,

$$
\sum_{|n| \geq n_{0}}\left|\widehat{g}(n) z^{n}\right| \leq \sum_{|n| \geq n_{0}}|\widehat{g}(n)|^{p}|z|^{n} \leq \sum_{|n| \geq n_{0}}|\widehat{g}(n)|^{p} \omega(n)<\infty .
$$

It is routine to check that $\varphi_{z}$ is a complex homomorphism on $A_{p}(\omega)$. Thus $\varphi_{z} \in \triangle\left(A_{p}(\omega)\right)(z \in \Gamma(\omega))$. Let $\varphi \in \triangle\left(A_{p}(\omega)\right)$. Then $\|\varphi\| \leq 1$. Let $e_{n}(z)=$ $z^{n}(n \in \mathbb{Z}, z \in \Gamma)$. So $\left|\varphi\left(e_{n}\right)\right| \leq\left\|e_{n}\right\|_{p, \omega}=\omega(n)(n \in \mathbb{Z})$. Set $\varphi\left(e_{1}\right)=z_{0}$. Then, for each $n \in \mathbb{Z}, \varphi\left(e_{n}\right)=\varphi\left(e_{1}\right)^{n}=z_{0}^{n}$. It is clear that $\rho(2, \omega) \leq\left|z_{0}\right| \leq$ $\rho(1, \omega)$. So, for any $g \in A_{p}(\omega)$, we have

$$
\varphi(g)=\varphi\left(\sum_{n \in \mathbb{Z}} \widehat{g}(n) e_{n}\right)=\sum_{n \in \mathbb{Z}} \widehat{g}(n) \varphi\left(e_{n}\right)=\sum_{n \in \mathbb{Z}} \widehat{g}(n) z_{0}^{n}=\varphi_{z_{0}}(g) .
$$

Thus $\varphi=\varphi_{z_{0}}$. This quickly gives the desired homeomorphism and establishes our claim. Thus each function $g \in A_{p}(\omega)$ extends uniquely as an element (denoted by $g$ itself) in the set $B(\omega)$ consisting of all continuous functions on $\Gamma(\omega)$ which are holomorphic in the interior of $\Gamma(\omega)$. Now the construction of desired weights $\nu$ and $\chi$ is exactly as in [2].

Proof of Corollary 1. By the hypothesis, $\sum_{n \in \mathbb{N}}(\log \omega(n)) / n^{2}$ is convergent. Notice that $\sum_{n \geq 2} 1 /(n \log n)$ is divergent. For infinitely many $n \in \mathbb{N}$,

$$
\frac{\log \omega(n)}{n^{2}} \leq \frac{1}{n \log n}
$$

hence $\inf \{(\log \omega(n)) / n: n \in \mathbb{N}\}=0$, and $\rho(1, \omega)=1$. Similarly, $\rho(2, \omega)=1$. Then, from Theorem 1(I), $\nu=\omega$; and the result follows.

Proof of Theorem 2. (i) $\Rightarrow$ (ii). We prove that if $G$ is not discrete, then $L^{p}(G, \omega)$ is not closed under convolution. Let $V$ be a symmetric, open subset of $G$ containing the identity of $G$ such that $\bar{V}$ is compact. Since $\omega$ is continuous on $G$,

(a) $m_{\omega}\left(V^{2}\right)=\int_{G} \chi_{V^{2}}(t) \omega(t) d m(t)<\infty$.

Now choose a sequence $\left(V_{n}\right)$ of measurable subsets of $G$ such that

(b) $m_{\omega}\left(V_{n}\right)>0$;

(c) $V_{n} \subseteq V$;

(d) $V_{i} \cap V_{j}=\emptyset$ whenever $i \neq j$.

From the properties (a), (c) and (d), we have $\sum_{n=1}^{\infty} m_{\omega}\left(V_{n}\right)<\infty$. We may assume that $m_{\omega}\left(V_{n}\right)<2^{-n}(n \in \mathbb{N})$. Define $f, g: G \rightarrow \mathbb{C}$ as

$$
f=\chi_{V^{2}} \quad \text { and } \quad g=\sum_{n=1}^{\infty}\left[m_{\omega}\left(V_{n}\right) n^{2}\right]^{-1 / p} \chi_{V_{n}} .
$$


Then $\|f\|_{p, \omega}=m_{\omega}\left(V^{2}\right)<\infty$ and

$$
\|g\|_{p, \omega}=\sum_{n=1}^{\infty}\left[m_{\omega}\left(V_{n}\right) n^{2}\right]^{-1} \int_{G} \chi_{V_{n}}(t) \omega(t) d m(t)=\sum_{n=1}^{\infty} n^{-2}<\infty .
$$

Thus $f, g \in L^{p}(G, \omega)$. On the other hand,

$$
(f \star g)(t)=\int_{G} f\left(s^{-1} t\right) g(s) d m(s)=\int_{t V^{2}} g(s) d m(s) .
$$

If $t \in V$, then $t V^{2} \subseteq V$ and so

$$
(f \star g)(t)=\sum_{n=1}^{\infty}\left[m_{\omega}\left(V_{n}\right) n^{2}\right]^{-1 / p} m\left(V_{n}\right)=\sum_{n=1}^{\infty}\left[m_{\omega}\left(V_{n}\right) n^{2}\right]^{-1 / p} m_{\omega}\left(V_{n}\right) \frac{m\left(V_{n}\right)}{m_{\omega}\left(V_{n}\right)} .
$$

Since $\omega$ is bounded on compact subsets of $G$, there exists $K>0$ such that $\omega(t) \leq K(t \in V)$. Now

$$
m_{\omega}\left(V_{n}\right)=\int_{V_{n}} \omega(t) d m(t) \leq \int_{V_{n}} K d m(t)=K m\left(V_{n}\right) .
$$

Thus

$$
\frac{m\left(V_{n}\right)}{m_{\omega}\left(V_{n}\right)} \geq \frac{1}{K} \quad(n \in \mathbb{N}) .
$$

Therefore

$$
(f \star g)(t) \geq \frac{1}{K} \sum_{n=1}^{\infty} 2^{n(1 / p-1)} n^{-2 / p}=\infty .
$$

Hence $f \star g$ cannot be in $L^{p}(G, \omega)$ as it becomes infinite on a set $V$ of positive measure.

(ii) $\Rightarrow$ (i). If $G$ is discrete, then it is easy to verify that $L^{p}(G, \omega)$ is closed under convolution.

(ii) $\Rightarrow$ (iv). Let $G$ be discrete. Let $f, g \in L^{p}(G, \omega)$ and $f \neq g$. Therefore $f(t) \neq g(t)$ for some $t \in G$. Define $\varphi_{t}: L^{p}(G, \omega) \rightarrow \mathbb{C}$ as $\varphi_{t}(h)=h(t)(h \in$ $\left.L^{p}(G, \omega)\right)$. It is easy to verify that $\varphi_{t}$ is a continuous linear functional on $L^{p}(G, \omega)$. Also $\varphi_{t}(f)=f(t) \neq g(t)=\varphi_{t}(g)$. Thus (iv) follows.

$(\mathrm{v}) \Leftrightarrow(\mathrm{vi})$. Since $\omega$ is continuous, $E \subset G$ is an $m_{\omega}$-atom if and only if $E$ is $m$-atom; and due to left translation invariance of $m$, this happens if and only if $t E$ is an atom for any $t \in G$.

(iii) $\Rightarrow\left(\right.$ vi). Let $L^{p}(G, \omega)$ have a non-zero continuous linear functional. Then, by [10, Corollary 4.2.3], $G$ admits an atom, say $E$. Since the Haar measure on $G$ is left translation invariant, we may assume that $E$ contains the identity $e$ of $G$.

Next we claim that every set of positive measure contains an atom (measure-theoretically). Let $F \subset G$ be of positive measure. If $F=G$ a.e. $m$, we are done. Let $m(G \backslash F)>0$. Now, either $F \cap E=\emptyset$ a.e. $m$ or $m(F \cap E)>0$. Since $E$ is an atom, in the latter case $F \cap E=E \subset F$ a.e. $m$. Suppose that 
$F \cap E=\emptyset$ a.e. $m$. Then $G \backslash F=E$ a.e. $m$. Now, for any $t \in G$, either $t E \cap E=\emptyset$ or $t E=E$ a.e. $m$. If $t E=E$ for all $t \in G$, then $G=E$ a.e. $m$, which is not possible as $m(F)=m(G \backslash E)>0$. Therefore there exists $t \in G$ such that $t E \cap E=\emptyset$ a.e. $m$. Then $t E \subset G \backslash E=F$ a.e. $m$. Thus $t E$ is an atom contained in $F$. This proves our claim. Now $G$ is a union of its atomic part, which is a union of atoms, and a non-atomic part. In this case the measure of the non-atomic part is zero; otherwise it will contain an atom. Thus (vi) follows.

$(\mathrm{v}) \Rightarrow$ (ii). Suppose that $G$ admits an atom, say $E$. We may assume that $e \in E$. First we assert that $E$ is an atom if and only if $E^{-1}$ is an atom. Since $m$ is left invariant, it follows from $[6,2.32$, p. 48] that $m(E)=0$ if and only if $m\left(E^{-1}\right)=0$. Let $F \subset E^{-1}$ be measurable. Then $F^{-1} \subset E$ is measurable. If $m(F)>0$, then $m\left(F^{-1}\right)>0$. Since $F^{-1} \subset E$, we have $F^{-1}=E$. Therefore $F=E^{-1}$. This proves our assertion.

Now define $g=m\left(E^{-1}\right)^{-1} \chi_{E}$. Then $g \in L^{1}(G)$. We claim that $g$ is the identity of $L^{1}(G)$. Let $f \in L^{1}(G)$ and $t \in G$. Then

$$
\begin{aligned}
(f \star g)(t) & =\int_{G} f\left(s^{-1} t\right) g(s) d m(s)=\frac{1}{m\left(E^{-1}\right)} \int_{E} f\left(s^{-1} t\right) d m(s) \\
& =\frac{1}{m\left(E^{-1}\right)} \int_{t E^{-1}} f(s) d m(s) .
\end{aligned}
$$

Note that $t E^{-1}$ is an atom, $t \in t E^{-1}$, and every measurable function is constant on an atom. Therefore

$$
(f \star g)(t)=\frac{1}{m\left(E^{-1}\right)} f(t) \int_{t E^{-1}} d m(s)=f(t) .
$$

Hence $f \star g=f$; similarly, $g \star f=f$. Thus $g$ is the identity of $L^{1}(G)$. It follows that $G$ is discrete.

(vi) $\Rightarrow$ (iv). Assume that $G$ admits sufficiently many atoms. Now let $f, g \in$ $L^{p}(G, \omega)$ be such that $f \neq g$. Then there exists an atom, say $E$, on which $f \neq g$. Also since every measurable function on an atom is constant, $f=$ $c_{f} \neq c_{g}=g$ on $E$. Define $\varphi_{E}: L^{p}(G, \omega) \rightarrow \mathbb{C}$ as

$$
\varphi_{E}(h)=\int_{E} h(s) d m_{\omega}(s) \quad\left(h \in L^{p}(G, \omega)\right) .
$$

Then $\varphi_{E}$ is a continuous linear functional on $L^{p}(G, \omega)$. Moreover, $\varphi_{E}(f)=$ $m_{\omega}(E) c_{f} \neq m_{\omega}(E) c_{g}=\varphi_{E}(g)$. Thus (iv) follows.

(i) $\Rightarrow$ (vi). Since $G$ is discrete, it is clear that $G$ is a union of atoms.

(iv) $\Rightarrow$ (iii) is clear.

Since $G$ is discrete, $L^{p}(G, \omega)=\ell^{p}(G, \omega)$. If $\omega$ is symmetric, then $\ell^{p}(G, \omega)$ is a *-subalgebra of $\ell^{1}(G)$. Since $\ell^{1}(G)$ admits a $C^{*}$-norm, it is an $A^{*}$-algebra. So, by [9, Theorem 4.1.19], $\ell^{p}(G, \omega)$ is semisimple. If $G$ is abelian, then 
$\ell^{p}(G, \omega)$ is a subalgebra of the commutative Banach algebra $\ell^{1}(G, \omega)$. Since $\ell^{1}(G, \omega)$ is semisimple [1], $\ell^{p}(G, \omega)$ is semisimple [9, Corollary 2.3.7].

Acknowledgements. The authors are grateful to the referee for giving fruitful suggestions. The work has been supported by the UGC-SAP-DRS grant No. F.510/5/DRS/2004(SAP-I) provided to the Department of Mathematics, Sardar Patel University.

\section{References}

[1] S. J. Bhatt and H. V. Dedania, A Beurling algebra is semisimple: An elementary proof, Bull. Austral. Math. Soc. 66 (2002), 91-93.

[2] -, -, Beurling algebra analogues of the classical theorems of Wiener and Lévy on absolutely convergent Fourier series, Proc. Indian Acad. Sci. (Math. Sci.) 113 (2003), 179-182.

[3] H. G. Dales and A. T.-M. Lau, The second duals of Beurling algebras, Mem. Amer. Math. Soc. 177 (2005), no. 836.

[4] Y. Domar, Harmonic analysis based on certain commutative Banach algebras, Acta Math. 96 (1956), 1-66.

[5] R. E. Edwards, Fourier Series, Vol. II, Holt, Rinehart and Winston, New York, 1967.

[6] G. Folland, A Course in Abstract Harmonic Analysis, CRC Press, 1995.

[7] I. M. Gel'fand, D. Raı̆kov and G. E. Šilov, Commutative Normed Rings, Chelsea Publ., New York, 1964.

[8] P. Lévy, Sur la convergence absolue des séries de Fourier, Compos. Math. 1 (1935), $1-14$.

[9] C. E. Rickart, General Theory of Banach Algebras, Van Nostrand, Princeton, NJ, 1960 .

[10] S. Rolewicz, Metric Linear Spaces, Reidel and PWN-Polish Sci. Publ., 1985.

[11] —, On multi-dimensional generalizations of the Wiener-Żelazko and Lévy-Żelazko theorems, in: Topological Algebras, their Applications, and Related Topics, K. Jarosz and A. Sołtysiak (eds.), Banach Center Publ. 67, Inst. Math., Polish Acad. Sci., 2005, 283-291.

[12] N. Wiener, Tauberian theorems, Ann. of Math. 33 (1932), 1-100.

[13] W. Żelazko, Selected Topics in Topological Algebras, Lecture Notes Ser. 31, Aarhus Univ., 1971.

Department of Mathematics

Sardar Patel University

Vallabh Vidyanagar-388 120

Gujarat, India

E-mail: subhashbhaib@yahoo.co.in

lightatinfinite@gmail.com

hvdedania@yahoo.com 